\title{
Distribution of drug-resistant bacteria and rational use of clinical antimicrobial agents
}

\author{
CHENLIANG ZHOU ${ }^{1}$, XIAOBING CHEN $^{2}$, LIWEN WU ${ }^{1}$ and JING QU ${ }^{1}$ \\ ${ }^{1}$ Department of Orthopedic Surgery, The Fourth Affiliated Hospital of Harbin Medical University, Harbin, \\ Heilongjiang 150001; ${ }^{2}$ The People's Hospital in Altay Region, Altay, Xinjiang 836500, P.R. China
}

Received February 29, 2016; Accepted April 8, 2016

DOI: 10.3892/etm.2016.3239

\begin{abstract}
Open wound may lead to infection in patients. Due to overuse of medication, certain bacteria have become resistant to drugs currently available. The aim of the present study was to provide a guide to ameliorate the appropriate and rational use of clinical antimicrobial agents by analyzing the distribution of drug-resistant pathogenic bacteria in patients. Between October 2013 and January 2015, 126 patients were selected at the Department of Orthopedics. Wound secretion samples were collected, and the pathogen bacteria isolated and identified. Identification was performed using an automated identification instrument and the Kirby-Bauer antibiotic method was used to evaluate the bacterial resistance. Of the 126 patients, 118 patients were infected (infection rate, 93.65\%). Additionally, 47 strains of gram-positive pathogenic bacteria (39.83\%) and 71 strains of pathogenic-gram negative bacteria $(60.17 \%)$ were identified. The bacteria were most likely to be resistant to penicillin while sensitive to vancomycin and imipenem. Some bacteria were resistant to several antibacterial agents. The results showed that existing risk factors at the Department of Orthopedics were complex and any non-standard procedures were able to cause bacterial infection. There were obvious dissimilarities among infectious bacteria with regard to their sensitivity to various antibacterial agents. Manipulation techniques during the treatment process were performed in a sterile manner and the use of antibacterial agents was required to be strictly in accordance with the results of drug sensitivity tests to provide effective etiologic information and a treatment plan for clinical trials and to reduce the risk of infection by multi-resistant bacteria.
\end{abstract}

Correspondence to: Dr Jing Qu, Department of Orthopedic Surgery, The Fourth Affiliated Hospital of Harbin Medical University, 37 Yiyuan Street, Harbin, Heilongjiang 150001, P.R. China

E-mail: xxumqwbay509@163.com

Key words: drug-resistance, pathogenic bacteria, drug sensitivity test

\section{Introduction}

Infection is a common complication that occurs in clinical trials (1). Since the majority of patients of the Department of Orthopedics have open wounds, there is a high risk of infection for these patients. Infections may include complicated osteomyelitis, and can be serious and even life threatening (2). In recent years, due to overuse and misuse of antibiotics, certain pathogen bacteria have become resistant to certain drugs. The development of antibiotic-resistant bacteria and their distribution worldwide is the result of many years of constant selection from human overuse and misuse of antibiotics (2). Antibiotic resistance, particularly the problems associated with superbacteria, has become a major public health issue and a global impediment for medical workers and investigators in the 21 st century.

Since patients in the Department of Orthopedics are easily infected, antibacterial agents are key elements for the treatment of open wounds of patients (3-5). To analyze the distribution of drug-resistant pathogenic bacteria in infected patients and to provide a guide to ameliorate the appropriate use of antibiotics, we analyzed the distribution of drug-resistant bacteria in 126 patients at the Department of Orthopedics. Wound secretion samples were collected, the pathogen bacteria isolated and cultured, and consequently drug-resistance analyses were performed.

\section{Patients and methods}

General information. Between October 2013 and January 2015, 126 patients were selected at the Department of Orthopedics, The Fourth Affiliated Hospital of Harbin Medical University (Heilongjiang, China). There were 72 male and 48 female patients with an age range of 16-72 years and an average age of $39.4 \pm 10.2$ years. Of the 126 patients, 32 cases had infectious arthritis, 48 cases had lower limb open wound infection, 18 cases had soft issue abscess, 21 cases had postoperative infection and 7 cases had other types of infection.

Identification of bacteria and drug sensitivity test. Samples from the wound secretions and pus of patients were taken in a sterile manner and immediately sent for isolation and purification. The isolation and purification protocols were based on The National Clinical Test Regulation of 


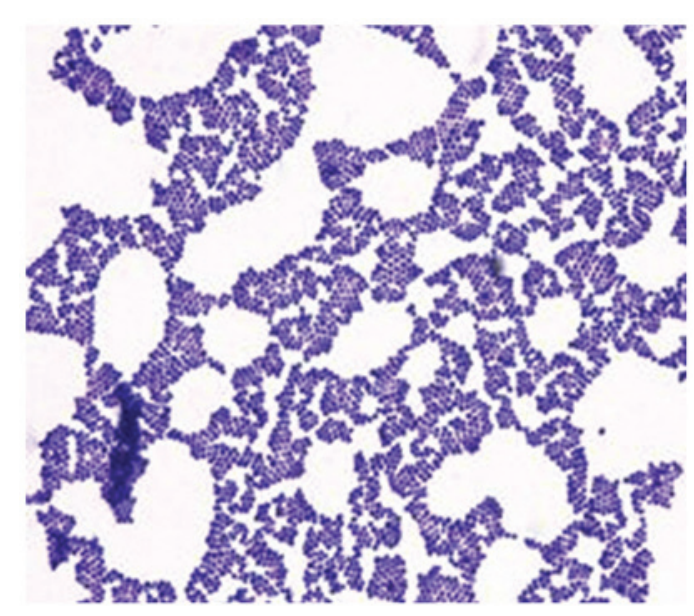

Figure 1. Staphylococcus aureus.

Operation (6). For the identification of pathogen bacteria an automated microorganism identification instrument was used. The Kirby-Bauer antibiotic testing agar diffusion method was used to evaluate bacterial resistance. In this process, Staphylococcus aureus ATCC25923 (Fig. 1), Pseudomonas aeruginosa ATCC27853, Escherichia coli ATCC25922 (Fig. 2) and Klebsiella pneumonia ATCC700603 were used as quality control bacteria.

Statistical analysis. Data were analyzed using SPSS 21.0 software (IBM SPSS, Armonk, NY, USA). A t-test was used to make comparisons between different groups. The size of the test was $\alpha=0.05$. $\mathrm{P}<0.05$ was considered to indicate a statistically significant difference.

\section{Results}

Infection rate. Of the samples isolated from 126 patients, 118 samples (infection rate, $93.65 \%$ ) had bacterial infections.

Pathogen distribution. Isolated pathogens included a variety of gram-positive and -negative bacteria, with gram-negative bacteria constituting the most abundant microorganisms. In total, 47 gram-positive (39.83\%) and 71 gram-negative $(60.17 \%)$ bacterial strains were identified. In the gram-positive group, Staphylococcus aureus, Staphylococcus epidermidis, Enterococcus sp. and Staphylococcus pyogenes were identified. In the gram-negative group Escherichia coli, Pseudomonas aeruginosa, Klebsiella pneumonia, and Enterobacter cloacae were identified. The most common bacteria were Pseudomonas aeruginosa, Staphylococcus aureus, Escherichia coli, Klebsiella pneumonia and Staphylococcus epidermidis (Table I).

Drug-resistant bacteria. The results of the drug resistance tests showed that several strains were resistant to multiple antibacterial agents. Pathogens such as Acinetobacter baumannii were resistant to the majority of antibacterial agents used in those tests. Gram-positive bacteria were sensitive to vancomycin (7), while almost all of the bacteria were entirely resistant to commonly used antibiotics, such as penicillin and erythromycin. Gram-negative bacteria
Table I. Isolated pathogens and their distribution.

\begin{tabular}{lcc}
\hline $\begin{array}{l}\text { Pathogen } \\
\text { classification }\end{array}$ & $\begin{array}{c}\text { No. of isolated } \\
\text { strains, strain }\end{array}$ & $\begin{array}{c}\text { Composition } \\
\text { ratio, \% }\end{array}$ \\
\hline Gram-positive bacteria & 47 & 39.83 \\
$\quad$ Staphylococcus aureus & 16 & 13.56 \\
Staphylococcus epidermidis & 12 & 10.16 \\
Enterococcus faecalis & 10 & 8.47 \\
Staphylococcus purulent & 6 & 5.08 \\
Staphylococcus haemolyticus & 4 & 3.39 \\
Gram-negative bacteria & 71 & 60.17 \\
Pseudomonas aeruginosa & 20 & 16.95 \\
Escherichia coli & 15 & 12.71 \\
Klebsiella pneumonia & 12 & 10.17 \\
Acinetobacter baumannii & 10 & 8.47 \\
Bacillus aerogenes & 5 & 4.24 \\
Enterobacter cloacae & 4 & 3.39 \\
Others & 5 & 4.24 \\
Total & 118 & 100.0 \\
\hline
\end{tabular}

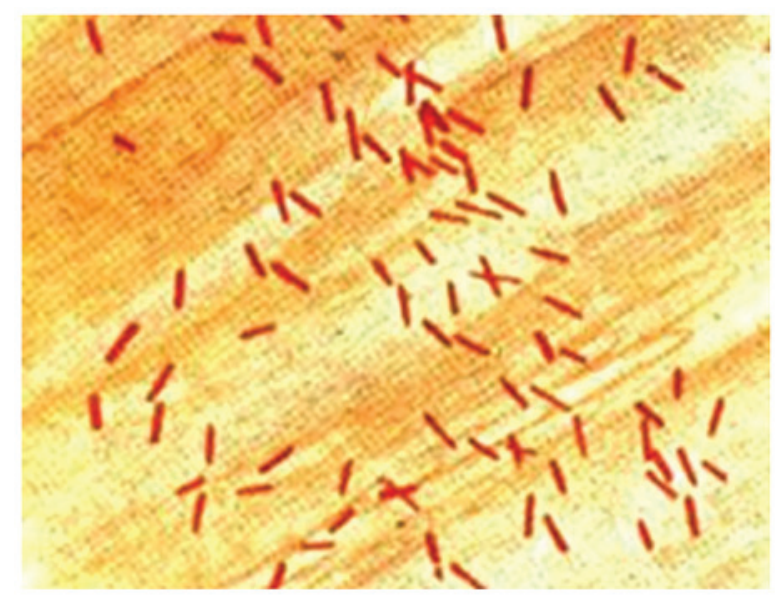

Figure 2. Escherichia coli.

were sensitive to meropenem and imipenem, while almost completely resistant to sulbactam and ampicillin. We selected typical gram-positive and -negative bacteria (Staphylococcus aureus, Staphylococcus epidermidis, Enterococcus faecalis, Pseudomonas aeruginosa, Escherichia coli, Klebsiella pneumonia and Acinetobacter baumannii) to test their resistance to antibacterial agents (Tables II and III).

\section{Discussion}

The overuse of antibiotics has led to the emergence of several drug-resistant bacteria that render the combat against bacterial infections more difficult. The harm caused by these drug-resistant bacteria has become more severe due to the lack of appropriate antibacterial agents in our arsenal (8). In order to achieve a better understanding regarding the drug resistance phenomenon, we need to expand our knowledge 
Table II. Drug-resistance test results for gram-positive bacteria [n (\%)].

\begin{tabular}{lccc}
\hline Antibacterial agents & Staphylococcus aureus 16 & Staphylococcus epidermidis 12 & Enterococcus faecalis 10 \\
\hline Penicillin, $\mathrm{n}(\%)$ & $14(87.5)$ & $9(75)$ & $8(80)$ \\
Erythromycin, $\mathrm{n}(\%)$ & $15(93.75)$ & $10(83.33)$ & $9(90)$ \\
Gentamicin, $(\%)$ & $4(25)$ & $8(66.67)$ & $7(70)$ \\
Rifampicin, $\mathrm{n}(\%)$ & $6(37.5)$ & $7(58.33)$ & $4(40)$ \\
Levofloxacin, $\mathrm{n}(\%)$ & $9(56.25)$ & $7(58.33)$ & $5(50)$ \\
Ciprofloxacin, $\mathrm{n}(\%)$ & $6(37.5)$ & $7(58.33)$ & $3(30)$ \\
Ampicillin/sulbactam, n (\%) & $8(66.67)$ & $4(40)$ \\
Amoxicillin, $\mathrm{n}(\%)$ & $10(62.5)$ & $8(66.67)$ & $4(40)$ \\
Cefazolin, $\mathrm{n}(\%)$ & $3(18.75)$ & $9(75)$ & $5(50)$ \\
Ceftriaxone, $\mathrm{n}(\%)$ & $4(25)$ & $8(66.67)$ & $4(40)$ \\
Vancomycin, $\mathrm{n}(\%)$ & $3(18.75)$ & 0 & 0
\end{tabular}

Table III. Drug-resistance test results for gram-negative bacteria [n (\%)].

\begin{tabular}{lcccc}
\hline Antibacterial agents & Pseudomonas aeruginosa & Escherichia coli & klebsiella pneumonia & Acinetobacter baumannii \\
\hline Sulbactam, n (\%) & $18(90)$ & $9(60)$ & $8(66.67)$ & $8(80)$ \\
Ampicillin, n (\%) & $19(95)$ & $10(66.67)$ & $9(75)$ & $9(90)$ \\
Gentamicin, n (\%) & $7(35)$ & $8(53.33)$ & $7(58.33)$ & $4(40)$ \\
Ceftazidime, n (\%) & $9(45)$ & $7(46.67)$ & $4(33.33)$ & $4(40)$ \\
Cefepime, n (\%) & $12(60)$ & $7(46.67)$ & $5(41.67)$ & $2(20)$ \\
Ceftriaxone, n (\%) & $9(45)$ & $7(46.67)$ & $3(25)$ & $3(30)$ \\
Cefazolin, n (\%) & $13(65)$ & $8(53.33)$ & $4(33.33)$ & $2(20)$ \\
Ciprofloxacin, n (\%) & $6(30)$ & $8(53.33)$ & $4(33.33)$ & $5(50)$ \\
Norfloxacin, n (\%) & $7(35)$ & $8(60)$ & $5(41.67)$ & $4(40)$ \\
Levofloxacin, n (\%) & $6(30)$ & $4(53.33)$ & $4(33.33)$ & $4(40)$ \\
Meropenem, n $(\%)$ & $1(5)$ & 0 & 0 & 0 \\
Imipenem & 0 & 0 & 0 & 0 \\
\hline
\end{tabular}

of the proper use of antibacterial agents (9). We investigated the occurrence of drug-resistant bacteria in patients at the Department of Orthopedics and analyzed the distribution of pathogens and antibacterial agents. We successfully isolated bacterial pathogens from 118 patients, and identified that those patients were primarily infected with Pseudomonas aeruginosa, Escherichia coli and Klebsiella pneumoniae. Of the gram-positive bacteria, Staphylococcus aureus, Staphylococcus epidermidis and Enterococcus faecalis had the highest infection rates. These results were consistent with the existing literature (10). Drug sensitivity test results revealed that most gram-negative bacteria were not sensitive to sulbactam and ampicillin, but were sensitive to meropenem and imipenem. Gram-negative bacteria were resistant to penicillin and erythromycin, but sensitive to vancomycin (11). Drug resistance continuously increased (12-14), thus, the work of clinical workers became more cumbersome.

Results obtained from previous studies (15-18) revealed that: i) Most patients had open wounds that were easily infected; ii) external wounds caused immune disorders, thereby causing alterations in intestinal flora and leading to internal infections; iii) errors during clinical treatment, non-sterile manipulation and the failure to treat the wounds in a timely manner potentially increased the risk of infection; and iv) failure to sterilize the wound also increased the risk of infection.

Since their identification, antibiotics have been crucial agents in the battle against infectious diseases caused by bacteria. Antimicrobial therapy has been an important reason for the significant increase of average life expectancy in the 20th century (19). The increase of drug-resistant bacteria poses a serious threat to public health and the economy. The emergence of superbacteria has indicated that the available pool of antibacterial agents are to be appropriately used and that the appropriate antibacterial agent to which the pathogens are sensitive should be utilized (19). We should only consider the use of newer and more potent antibacterial agents in severe infections caused by multi-resistant bacteria.

The most effective manner in which to prevent infection is thorough correct debridement prior to surgery and timely sterilization thereafter (20). The use of antibiotics should be guided by patients' etiological tests (21).

In conclusion, we identified that the existing risk factors in the Department of Orthopedics were complex and any non-standard procedures may cause bacterial infection. 
Additionally, there were evident dissimilarities among infectious bacteria with regard to their sensitivity to various antibacterial agents. Manipulation techniques during the treatment process are to be performed in a sterile manner and the use of antibacterial agents should be strictly in accordance with the results of drug sensitivity tests to provide effective etiologic information and a treatment plan for clinical trials and to reduce the risk of infection by multi-resistant bacteria.

\section{References}

1. Dong L, Zhang XY, Liu ZY, Jiang FL, Zhang J and Wu YP. Analysis of Distribution and Drug Resistance of Pathogens in the Infection Inpatients from Orthopedic Department of Our Hospital during 2011-2013. China Pharm 2: 195-197, 2015.

2. Ramos-Luces O, Molina-Guillén N, Pillkahn-Díaz W, Moreno-Rodríguez J, Vieira-Rodríguez A and Gómez-León J: Surgical wound infection in general surgery. Cir Cir 79: 323-329, 2011.

3. Wu XY, Ni KX and Li SB: Clinical characteristics and resistance of wound surface infections causing by Bacillus cereus in the department of orthopaedics. Zhongguo Gu Shang 26: 753-756, 2013 (In Chinese).

4. Bouza E, Burillo A, Munoz P, Cercenado E and RodríguezCréixems M: Semiquantitative culture of open surgical wounds for diagnosis of surgical site infection. Eur J Clin Microbio Infect Dis 23: 119-122, 2004.

5. Wysocki AB: Evaluating and managing open skin wounds: Colonization versus infection. AACN Clin Issues 13: 382-397, 2002.

6. Zhao X: Pathogen analysis and clinical prevention and treatment of inhospital postoperative infection of aging patients in department of orthopedics. Practical Clinical Medicine 19: 145-147, 2015 (In Chinese)

7. Bagga B and Shenep JL: Management of infections caused by vancomycin-resistant gram-positive bacteria. Pediatr Infect Dis J 29: 662-664, 2010.

8. O'Neill AJ: New antibacterial agents for treating infections caused by multi-drug resistant Gram-negative bacteria. Expert Opin Investig Drugs 17: 297-302, 2008.

9. Ii K, Ichikawa S, Al-Dabbagh B, Bouhss A and Matsuda A: Function-oriented synthesis of simplified caprazamycins: Discovery of oxazolidine-containing uridine derivatives as antibacterial agents against drug-resistant bacteria. J Med Chem 53 3793-3813, 2010.
10. Li S and Ye G: Infection distribution of Aureus and analysis on its drug resistance. J Chin Hosp Infect 20: 3045-3046, 2010.

11. Qiubao A and Aimin H: Clinical distribution and drug resistance analysis of pseudomonas aeruginosa in our hospital. Med Infant 13: 236-237, 2013.

12. Maltezou HC, Giakkoupi P, Maragos A, Bolikas M, Raftopoulos V, Papahatzaki H, Vrouhos G, Liakou V and Vatopoulos AC: Outbreak of infections due to KPC-2-producing Klebsiella pneumoniae in a hospital in Crete (Greece). J Infect 58: 213-219, 2009.

13. Gregory CJ, Llata E, Stine N, Gould C, Santiago LM, Vazquez GJ, Robledo IE, Srinivasan A, Goering RV and Tomashek KM: Outbreak of carbapenem-resistant Klebsiella pneumoniae in Puerto Rico associated with a novel carbapenemase variant. Infect Control Hosp Epidemiol 31: 476-484, 2010.

14. Meunier O: From the discovery of antibiotics to emerging highly drug-resistant bacteria. Soins 797: 14-20, 2015 (In French).

15. Selasi GN, Nicholas A, Jeon H, Lee YC, Yoo JR, Heo ST and Lee JC: Genetic basis of antimicrobial resistance and clonal dynamics of carbapenem-resistant Acinetobacter baumannii sequence type 191 in a Korean hospital. Infect Genet Evol 36: $1-7,2015$.

16. Kirkland KB, Briggs JP, Trivette SL, Wilkinson WE and Sexton DJ: The impact of surgical-site infections in the 1990s: Attributable mortality, excess length of hospitalization, and extra costs. Infect Control Hosp Epidemiol 20: 725-730, 1999.

17. Olsen MA, Nepple JJ, Riew KD, Lenke LG, Bridwell KH, Mayfield J and Fraser VJ: Risk factors for surgical site infection following orthopaedic spinal operations. J Bone Joint Surg Am 90: 62-69, 2008.

18. Cole MR, Hobden JA and Warner IM: Recycling antibiotics into GUMBOS: A new combination strategy to combat multi-drug-resistant bacteria. Molecules 20: 6466-6487, 2015.

19. Pull ter Gunne AF, Hosman AJ, Cohen DB, Schuetz M, Habil D, van Laarhoven CJ and van Middendorp JJ: A methodological systematic review on surgical site infections following spinal surgery: part 1: risk factors. Spine 37: 2017-2033, 2012.

20. Olsen MA, Butler AM, Willers DM, Devkota P, Gross GA and Fraser VJ: Risk factors for surgical site infection after low transverse cesarean section. Infect Control Hosp Epidemiol 29: 477-484, discussion 485-486, 2008.

21. Ferronato AE, Gilio AE, Ferraro AA, Paulis M and Vieira SE: Etiological diagnosis reduces the use of antibiotics in infants with bronchiolitis. Clinics (Sao Paulo) 67: 1001-1006, 2012. 